sarcoma, the histological appearances were those of a "hard fibroma," and the long history and complete lack of metastases do not suggest a malignant neoplasm. In retrospect this was almost certainly the first account of the disease described some 10 years later by Riedel, and incidentally, of its association (and contiguity) with idiopathic mediastinal fibrosis.

Perhaps this tantalizing group of diseases, with its cumbersome and variable nomenclature, should be called "Bowlby's disease". -I am, etc.

\section{J. Mitchinson}

Department of Pathology,
University of Cambridge

1 Comings, D. E., Skubi, K. B., Van Eyes, J., and Motulsky, A. G., Annals of Internal 2 Mulvaney, W. P., Gordon, L. Z., and Gritti, E. J., Fournal of Urology, 1968,'99. 417 3 Ullmann, A. S., and Dacso, M. R., Diseases of the Chest, 1968, 53, 656.

4 Bowlby, A. A., Transactions of the Pathological Society of London, 1885, 36, 420 .

\section{Mental Health Aspects of Shoplifting}

SIR,-The paper on the mental aspects of shoplifting (11 September, p. 612) was of interest to magistrates as well as doctors.

It might be preferable if the older women were not brought to court. If there is a "not guilty" plea the bench has to decide on guilt before the probation officer's report is read. The usual defence is that there was no intent to steal, and that the defendant was unaware of her actions. The store detective's evidence often describes the furtive way articles are transferred to the private shopping bag, and it becomes diffcult not to convict the defendant. It may be that the behaviour is a cry for recognition or help, but a crime seems to have been committed. Psychiatrically, it may be naive to discuss whether she is "aware" of what she is doing and whether she has an "intent to steal," but the magistrate has to answer these questions.

Having found her guilty, what are the effects of sentence? Those usually considered are a straight fine, perhaps coupled with advice to see her doctor: this has the advantage of completing the affair rapidly, as the fine is usually able to be paid in seven days; or probation; or an absolute or conditional discharge, which may be contrary to local policy because of the lack of deterrent value. Has punishment any therapeutic value?

Is it the appearance in court, the conviction, or the sentence that has the traumatic effect, and have the authors any information about the results of different sentences on the individuals? - I am, etc.,

PETER TOMSON

Abbots Langley,

Herts

\section{Interpretation of Arteriography}

SIR,-May I make one comment on your leading article "Fibromuscular Hyperplasia of the Internal Carotid Artery" (11 September, p. 598)? In an otherwise clear exposition, with no less than 23 references from English and American literature, you say, in referring to the classic arteriographic picture of a "string of beads": "However, this angiographic appearance is insufficient evidence to allow a firm diagnosis of the disease to be made, for a similar picture may result from spasm of the carotid artery, particularly when related to a difficult puncture of the artery during arteriography. Surgical inspection and pathological examination of the vessel is the only absolute way of making the diagnosis." What do you mean by this? If an appearance is "classic," it is "classic." You do not quote any of your 23 authorities substantiating your caveat.

The arteriographic picture of spasm of the artery is quite different from the "string of beads" sign, which I would agree is not only classic but pathognomonic. What do you imply by a "difficult picture of the artery"? The puncture may certainly be difficult and three possibilities may ensue: (1) It is so difficult that it fails, in which case there is no arteriographic appearance. (2) If successful, the classic and pathognomonic sign will be revealed. (3) If the contrast substance enters the wall rather than the lumen of the artery, another pathognomonic sign will appear which closely simulates complete occlusion of the vessel, a now well-known artefactual appearance. ${ }^{1}$

I would strongly contest the statement that "surgical inspection" is "an absolute way of making the diagnosis." The surgeon cannot see the wall layers or shape of the lumen of the artery without making an incision. Certainly pathological examination is "an absolute way."

Where does the scepticism of some medical writers end in regard to radiology? It would be a reductio ad absurdum to ask a surgeon or pathologist to confirm or refute the presence of a fracture revealed on an $x$-ray film in order to make an "absolute diagnosis," but how does this example diffe in principle from the condition under discussion?-I am, etc.,

Lysholm Radiological Department,

JAMES BULL

Lysholm Radiological

Queen Square,

1 Newton, T. H., and Couch, R. S. C., Radiology,

\section{Starch Granulomatosis of the Peritoneum}

SIR,-May we, through your columns, report two cases of starch powder granulomatosis of the peritoneum similar to those reported by Mr. J. Neely and Dr. J. D Davies (11 September, p. 625).

Both patients developed symptoms of malaise and ill-defined abdominal pain with pyrexia three weeks after laparotomy. Both were reoperated on within three days of each other and there were exactly similar intra-abdominal findings in each case There was free intraperitoneal fluid, the greater omentum appeared inflamed, and there were many dense adhesions. Histological examination of biopsy specimens of the greater omentum showed there to be an intense inflammatory reaction with eosinophils and foreign body giant cells. The intraperitoneal fluid was sterile on culture in both cases and no acid-fast bacilli could be detected either by Ziehl-Neelsen staining or by guinea pig inoculation. The reaction appeared to be directed towards anisotropic periodic-acid-Schiff-positive material, which was likely to be starch powder granules.

The first case was a 43-year-old woman who had a cholecystectomy and operative cholangiogram for chronic cholecystitis and cholelithiasis on 26 February 1971. Twenty days later she developed right-sided abdominal pain and pyrexia and was readmitted to hospital. On examination she was tender on the whole of the right side of the abdomen, and there was some induration around the lower half of the cholecystectomy incision. It was thought that she had a wound infection. However, the white cell count was consistently normal, serum amylase normal, blood culture normal, and there was no evidence of a subphrenic abscess. There was a urinary tract infection with Proteus spp. and Str. faecalis and the aspartate transaminase and alanine transaminase were slightly raised. She was observed for 11 days, during which time the pulse gradually rose to 120 beats per minute, the temperature to $102^{\circ} \mathrm{F}\left(38.9^{\circ} \mathrm{C}\right)$, and she became quite ill. Laparotomy was performed on 2 April and 3 litres of clear sterile slightly green fluid were aspirated from the peritoneal cavity. There was no evidence of a subphrenic abscess or acute pancreatitis, but the greater omentum was inflamed and stuck to all of the surrounding organs. Biopsies of the greater omentum were taken and the wound closed with drainage. Postoperatively she developed pulmonary oedema and a collapsed right lower lobe; she became very ill and exhausted and required intermittent positive pressure respiration for three days. Eventually she made a satisfactory recovery.

The second case was a 28-year-old man who underwent a selective proximal vagotomy for an active duodenal ulcer on 9 March 1971. Twenty-two days later he became unwell with a loss of appetite, malaise, and abdominal pains. He was admitted to hospital three days later when he was found to have a swinging temperature with a normal white cell count, and again it was thought he had a wound infection. The abdominal pain increased, and so 27 days after his original laparotomy reoperation was performed. The peritoneal cavity contained 1 litre of straw coloured fluid, which was sterile, the greater omentum was inflamed, and there were many dense intraabdominal adhesions. Biopsies were taken for histological examination and the wound closed with drainage. He continued to have a hectic fever for the following 13 days but eventually made a satisfactory recovery.

We feel it is worth recording these two cases because both patients were ill and on general grounds required exploration. Had they not had a previous operation and simply presented de novo the diagnosis would have been of serious peritoneal inflammatory disease.-We are, etc.

\section{J. F. Colin C. WASTELI}

Surgical Unit,

Westminster Medical School

The Gordon Hospital,

London S.W.1

SIR,-For the past three years I have used dry surgical gloves without finding it necessary to add extra powder lubricant.

The main glove manufacturers in England, who incidentally have a large export trade, have told me that so far as they know surgeons in Britain are practically the only ones using extra powder. Each glove already has a fine coating of powder and the makers are at present trying to eliminate it as far as possible from the outer surface. I would 